\section{Association between the Skull Base and Maxillofacial Morphology in Adults}

\section{Abstract}

In this study, we investigated the influence of the anteroposterior diameter and basal angle of the skull base on the maxillofacial morphology. The research materials were lateral cephalograms of 30 adult female patients on the first examination who received orthodontic treatment at the Orthodontic Department of Kanazawa Medical University Hospital. The 20 measurement items below were set based on digital cephalograms acquired by the standard method. (1. N-S 2. S-Ba 3. $\angle \mathrm{N}-\mathrm{S}-\mathrm{Ba}$ 4. Ptm-ANS/NF 5. SN-palatal pl. 6. Go-Me 7. Ar-Me 8. Ar-Go 9. $\angle S N-M a n d . p l . ~ 10$. Gonial Angle 11. $\angle S N-O c c . p l .12$. N-Me 13. N-NF 14. Me-NF 15. S-NF 16. Go-NF 17. U1/NF 18. U6/NF 19. L1/Mand.pl. 20. L6/Mand.pl.).

Regarding the study method, firstly, analysis of variance was performed to confirm intra-individual variation of each of the data to investigate whether the measurement items can be compared using the data as a preliminary experiment. No significant difference was noted in intra-individual variation in any measurement item, confirming that inter-individual variation can be compared using the data.

Then, the correlations between the measured values of each measurement item of 1. N-S 2. S-Ba 3. $\angle \mathrm{N}-\mathrm{S}-\mathrm{Ba}$ and 17 measurement items were investigated.

Significant positive correlations were noted between N-S and Ar-Go and between $\angle \mathrm{N}$-S-Ba and Ptm-ANS $/ \mathrm{NF}$, suggesting that the mandibular ramus height increases as the length of the anterior cranial base [N-S] increases, and the anteroposterior diameter of the maxilla increases as the basal angle increases.

In the present study, significant positive correlations were noted between $\mathrm{N}-\mathrm{S}$ and $\mathrm{Ar}-\mathrm{Go}$ and between $\angle \mathrm{N}-\mathrm{S}-\mathrm{Ba}$ and Ptm and $-\mathrm{ANS} / \mathrm{NF}$, clarifying that the anteroposterior diameter and basal angle of the skull base influence the maxillofacial morphology based on the specific measurement items.

Keywords: Skull base; Maxillofacial morphology; Cephalogram; Significant difference

\section{Noboru Demura*, Miho Hasumoto and Taichi Demura}

\begin{abstract}
Orthodontic Division of Oral-Maxillo-Facial Surgery, Kanazawa Medical University, Ishikawa 920-0293, Japan
\end{abstract}

*Corresponding author: Noboru Demura

n-demura@kanazawa-med.ac.jp

Asociate Professor of Oral-Maxillo-Facial Surgery, Kanazawa Medical University, 1-1 Daigaku, Kahoku-gun, Uchinada, Ishikawa 920-0293, Japan.

Citation: Demura N, Hasumoto M, Demura T (2018) Association between the Skull Base and Maxillofacial Morphology in Adults. J Orthod Endod Vol.4 No.2:10

\section{Introduction}

In clinical orthodontics, it has been pointed out that the morphology [basal angle] and anteroposterior diameter of the skull base influence the maxillofacial morphology including the positions and morphologies of the maxilla and mandible, but many points have not been elucidated. The relationship between the basal angle and malocclusion was pointed out in some reports but it was negative in many other reports. In this study, we investigated the influence of the anteroposterior diameter and basal angle of the skull base on the maxillofacial morphology.

\section{Research Materials}

The research materials were lateral cephalograms of 30 adult female patients on the first examination who received orthodontic treatment at the Orthodontic Department of Kanazawa Medical University Hospital. Each subject was confirmed to have no bad habits, such has abnormal swallowing, abnormal tongue morphology, such as macroglossia, pathological hypertrophy of the pharyngeal and palatine tonsil, or past medical history of otolaryngologic disease or surgery by an interview and visual examination. No patient with a congenital malformation, such 
as cleft palate and cleft lip, was included. The 20 measurement items below Table 1 were set based on digital cephalograms acquired by the standard method.

Table 1: The measurement items and significant difference in each measurement item (* indicates significant difference at a significance level of $5 \%$ ).

\begin{tabular}{|c|c|}
\hline \multicolumn{2}{|c|}{ Significant difference } \\
\hline 1 & $\mathrm{~N}-\mathrm{S}$ \\
\hline 2 & S-Ba \\
\hline 3 & $\angle \mathrm{N}-\mathrm{S}-\mathrm{Ba}$ \\
\hline 4 & Ptm-Ans /NF \\
\hline 5 & SN-palatal pl. \\
\hline 6 & Go-Me \\
\hline 7 & Ar-Me \\
\hline 8 & Ar-Go \\
\hline 9 & $\angle \mathrm{SN}-$ Mand.pl. \\
\hline 10 & Gonial Angle \\
\hline 11 & $1 \angle \mathrm{SN}-\mathrm{Occ}$.pl \\
\hline 12 & $\mathrm{~N}-\mathrm{Me}$ \\
\hline 13 & $\mathrm{~N}-\mathrm{NF}$ \\
\hline 14 & Me-NF \\
\hline 15 & S-NF \\
\hline 16 & Go-NF \\
\hline 17 & U1/NF \\
\hline 18 & U6/NF \\
\hline 19 & L1/Mand.pl. \\
\hline 20 & L6/Mand.pl. \\
\hline
\end{tabular}

Regarding the study method, firstly, analysis of variance was performed to confirm intra-individual variation of each of the data to investigate whether the measurement items can be compared using the data as a preliminary experiment. No significant difference was noted in intra-individual variation in any measurement item, confirming that inter-individual variation can be compared using the data. Then, the correlations between the measured values of each measurement item of $\mathrm{N}-\mathrm{S}, \mathrm{S}-\mathrm{Ba}, \angle \mathrm{N}$ S-Ba and 4-20 were investigated.

\section{Results}

Table 1 shows the results [the measurement items and significant difference in each measurement item]. Significant positive correlations were noted between N-S and Ar-Go and between Ar-Go and Ptm-ANS/NF, suggesting that the mandibular ramus height increases as the length of the anterior cranial base [ $\mathrm{N}$ $\mathrm{S}]$ increases, and the anteroposterior diameter of the maxilla increases as the basal angle increases.

\section{Discussion}

Ford [1] reported that the growth pattern of the anterior cranial base is intermediate between the nerve and general types, but individual bones forming the anterior cranial base follow the growth pattern of the nerve or general type without the intermediate type. It is considered that growth and development of the skull base are closely related to growth and development of the maxilla and mandible.

The anteroposterior diameter of the skull base increases through cartilage growth in spheno-ethmoidal synchondrosis, intersphenoidal synchondrosis, and sphenooccipital synchondrosis. Growth of intersphenoidal synchondrosis stops at birth and growth of spheno-ethmoidal synchondrosis completes at about 7 years old. Growth of sphenooccipital synchondrosis is important and ossification in the cartilage continues until near 20 years old. The amount of growth in this region is the largest, being the center of skull base growth [2].

When synchondrosis ossifies early, the cranial base remains short and midface or maxillary hypogrowth occurs. Accordingly, clinically, many cases show a type of anterior cross-bite. When the growth and development of synchondrosis are markedly suppressed, cranial dysplasia may occur and clavicle cranial dysplasia [cleidocranial dysostosis] is the typical disease. The main symptoms of this disease are hypoplasia of the clavicle, skull, and teeth and delayed tooth replacement initially reported by Martin [3] in 1765, and Marie and Sainton [4] established its disease concept as an independent bone malformation syndrome in 1897. Kreiborg et al. [5] reported that since the clivus bends in cleidocranial dysostosis, the basal angle is small and the mandible is present in a protruded position.

In the present study, a positive correlation was noted between $\mathrm{N}-\mathrm{S}$ and $\mathrm{Ar}-\mathrm{Go}$ and Ar-Go increased as N-S increased, suggesting an association.

Regarding the skull base, consistence between the growth peak in adolescence and that of the forebrain skull base and involvement of the morphology and growth pattern of the hindbrain skull base in growth of the mandibular ramus height in late adolescence have been reported [6], being consistent with our findings. It has been pointed out that the basal angle receives genetic influence [7], and the relationship with malocclusion has also been pointed out. It is considered that the size of the posterior cranial base is increased by growth of synchondrosis with the sphenoid bone. Lathman [8] performed a study of the cranial base in subjects aged 2 months to 18 years in which sphero-occipital synchondrosis was divided into the sphenoid and occipital bone sites and the former and latter gre $w$ for 10 and 18 years, respectively. Brodie [9] stated that this synchondrosis grows until 21 years old. Regarding the role of sphero-occipital synchondrosis, Koski [10] reported that it is a process to adjust the cranial base necessary for growth of the brain and securing the upper airway. Dhopatkar et al. [11], Hopkin et al. [12], Dibbets [13] stated that the basal angle was large in Angle Class II and decreased in the order of Angle Class I and Angle Class III. Anderson et al. [14] reported that the basal angle was the largest in Angle Class II followed by Angle Class III and then Angle Class I. Hopkin et al. [15] stated that the cranial base morphology and cranial base angle increased in the order of Angle Class III, Angle 
Class II, and Angle Class I. Jarvinen [16] reported that the Ar-S-N angle in Angle Class II was larger than that in Angle Class III. We observed a significant positive correlation between $\angle \mathrm{N}-\mathrm{S}-\mathrm{Ba}$ and Ptm-ANS / NF, suggesting that the anteroposterior diameter of the maxilla increases as the basal angle spreads, which indicates the association with malocclusion. On the other hand, Hildwein et al. [17] reported that $\angle B a-S-N$ did not differ between II and I, and Varjanne et al. [18] reported that no association was noted between the skull base length and basal angle. Similarly, Renfroe [19], Menezes [20], Guyer et al. [21] reported that the influence of the basal angle on malocclusion is small.

Regarding the association between the cranial base and maxillofacial morphology, Enlow and McNamara [22] reported that the basal angle is dilated in dolichocephaly and the mandible is present in a retruded position in many cases, whereas in brachycephaly, the basal angle is small and the mandible is present in a protruded position. Tulley and Campbell [23] reported changes in growth and development of the basal angle, in which the angle slightly dilates until adulthood is reached. The difference in the vertical morphology of the anterior cranial base influences not only the maxillary complex but also the mandible through being accompanied by dilation of the mandibular gonial angle and short or long mandibular ramus. In addition, the size and morphology of the mandible are associated with the functions of attaching muscles. It is considered that the masseter controls the morphology of the posterior margin of the mandibular ramus, lateral side of the mandibular angle, and mandibular angle. Throckmorton [24] stated that there are clear differences in the masseter between the long and short faces. Therefore, the mandibular morphology is influenced by not only the vertical morphology of the anterior cranial base but also attaching masticatory muscles. We are planning to investigate the maxillofacial morphology in consideration of the attachment and function of muscles around the oral cavity, such as the masticatory muscles.

\section{Conclusion}

In the present study, significant positive correlations were noted between N-S and Ar-Go and between $\angle \mathrm{N}-\mathrm{S}-\mathrm{Ba}$ and Ptm and -
ANS/NF, clarifying that the anteroposterior diameter and basal angle of the skull base influence the maxillofacial morphology based on the specific measurement items. We are planning to report this in more detail in the future.

\section{Declarations}

\section{Ethics approval and consent to participate}

This study was performed in accordance with the Declaration of Helsinki, and carried out with the approval of the Ethics Committee of the Kanazawa Medical University.

\section{Consent to publish}

Not applicable.

\section{Availability of data and materials}

The datasets used and/or analyzed during the current study are available from the corresponding authors on reasonable request.

\section{Competing interests}

The authors declare that they have no competing interests.

\section{Funding}

No funding has been received for this study.

\section{Authors' Contributions}

The roles of the authors were: ND, MH, TD contributed to the conception and design of the study, analysis and interpretation of data.

ND wrote the paper, $\mathrm{MH}$ and TD have critically read and edited the paper.

All authors have read and approved the final manuscript.

\section{Acknowledgement}

Not applicable.

\section{Conflict of Interest}

The authors declare that they have no conflict interest. 


\section{References}

1 Ford EHR (1958) Growth of the human cranial base. Am J Orthod Dentofac Orthop 44: 498-506.

2 Kisimoto T (1978) Orthodontia. Nihon lji Sinnposya. p: 9.

3 Martin M (1765) Sur un dep lacent natural de ia clvicule. J Med Chir Pharmacol 23: 456.

4 Mari e P, Sainton P (1897) Observation d'hydrocephalie hereditaire par vice de developpement du crane et du cerveau. Bull soc Med Hop Paris 14: 706.

5 Kreiborg S, Jensen BL, Bjork A, Skieller V (1981) Abnormalities of the cranial base in cleidocranial dysostosis. Am J Orthod 79: 549-557.

6 Ueda A (1991) A consideration on growth and development of the mandibular ramus in late adolescent period. J Japan Orthod Soc 50: 137-146.

7 Klocke A, Nanda RS, Kahl-Nieke B (2002) Role of cranial base flexure in developing sagittal jaw discrepancies. Am J Dentofac Orthop 122: 386-391.

8 Lathman RA (1972) The Sella point and postnatal growth of the human cranial base. Am J Orthod 61: 156-162.

9 Brodie AG (1941) On the growth pattern of the human head from the third month to the eight year of life. Am J Orthod 68: 209-267.

10 Koski K (1960) Some aspects of growth of the cranial base and the upper face. Orthodont Trans 68: 344-358.

11 Dhopatkar A, Bhatia S, Rock P (2002) An investigation into the relationship between the cranial base angle and malocclusion. Angle Orthod 72: 456-463.

12 Hopkin GB, Houston WJB, James GA (1968) The cranial base as an aetiological factor in malocclusion. Angle Orthod 38: 250-255.
13 Dibbets JM (1996) Morphological associations between the Angle Classes. Eur J Orthod 18: 111-118.

14 Anderson D, Popovich F (1983) Relation of cranial base flexure to cranial form and mandibular position. Am J Phys Anthoropol 61: 181-187.

15 Hopkin GB (1966) Craniofacial pattern in Mesio-occlusion. Am J Orthod 52: 927-928.

16 Jarvinen S (1980) Relation of the SNA angle to the saddle angle. Am J Orthod 78: 670-673.

17 Hildwein M, Bacon W, Turlot JC, Kuntz M (1986) Specificiteset discriminants mmajeurs dans une population de angle class II division1. Rev Orthop Dento Faciale 20: 197-208.

18 Varjanne I, Koshi K (1982) Cranial base, sagittal jaw relationship and occlusion: A radiological-craniometric appraisal. Proc Finn Dent Soc 78: 179-183.

19 Renfroe EW (1948) A study of the facial patterns associated with Class I, Class II division1, and Class II division2 malocclusions. Angle Orthod 18: 12-15.

20 Menezes DM (1974) Comparisons of craniofacial features of English children with Angle Class II division1 and Angle Class I malocclusions. J Dent 2: 250-254.

21 Guyer EC, Ellis EE, McNamara J, Behrrents RG (1986) Components of Class III malocclusion in juveniles and adolescents. Angle Orthod 56: 7-30.

22 Enlow DH, McNamara JA (1973) The neurocranial basis for facial form and pattern. Angle Orthodont 43: 256-270.

23 Tulley WJ, Campbell AC (1970) A manual of practical orthodontics. $3^{\text {rd }}$ edn. John Wright and Sons Ltd, Bristol. pp: 4-20.

24 Throckmorton GS, Finn RA, Bell WH (1980) Biomechanics of differences in lower facial height. Am J Orthod 77: 410-420. 